\title{
Formelkompromisse ohne klare Linie
}

\section{Ein kritischer Kommentar zur Sozialpolitik der Großen Koalition}

Dass die Vorschusslorbeeren, mit denen man Angela Merkel bei ihrem Amtsantritt als Bundeskanzlerin und nach ihren ersten öffentlichen Auftritten vor allem auf diplomatischem Parkett bedachte, schnell welkten und die Große Koalition ihren Kredit bei der Bevölkerungsmehrheit im Rekordtempo verspielte, dürfte mit der Art und Weise zu tun haben, wie sie den rot-grünen Reformkurs im Sozialbereich noch verschärfte und die Umverteilung von unten nach oben fortsetzte.

\section{Christoph Butterwegge}

Als Kanzler Gerhard Schröder bei der Bundestagswahl am 18. September 2005 abgewählt wurde, hätte sich für die SPD nach dem Um- bzw. Abbau des Sozialstaates durch die rot-grüne Koalition, für den die Kunstworte "Agenda 2010" und „Hartz IV" stehen, ${ }^{1}$ eine politische, programmatische und personelle Regeneration in der Opposition angeboten. So riet Rudolf Walther der Partei, sich zur „Selbstreflexion für einen sozialdemokratischen Neubeginn" genügend Zeit zu nehmen und nicht gleich wieder in Ministersessel zu steigen: „Wer regieren muss, das zeigen die sieben Jahre der rot-grünen Improvisationen, hat keine Zeit zum Nachdenken, sondern exekutiert Sachzwänge, treibt symbolische Politik oder delegiert das Nachdenken an externe Expertenklüngel, die dem parlamentarischen System den Garaus machen und die Parteien zu Marionetten herabstufen. ${ }^{22}$

Wer jedoch geglaubt hatte, die SPD werde sich nach der "Ära Schröder" von dessen politischem Kurs lösen und sich dieser ideologischen Erblast entledigen, sah sich getäuscht. Statt die nötige Vergangenheitsbewältigung zu leisten und neue Perspektiven zu entwickeln, zelebrierte der Karlsruher Parteitag vom 14. bis 16. November 2005 eine peinliche Abschieds- bzw. Nostalgieshow um und mit Gerhard Schröder. Dessen engste Vertraute in Parteiund Regierungsämtern machten weiterhin Karriere. So avancierte Frank-Walter Steinmeier, sein früherer Kanzleramtschef und Architekt der "Agenda 2010“, zum Außen- und der als nordrhein-westfälischer Ministerpräsident gescheiterte Peer Steinbrück zum Finanzminister der Großen Koalition. Trotz oder gerade wegen dieser Konti-

Prof. Dr. Christoph Butterwegge leitet die Abteilung für Politikwissenschaft an der Universität zu Köln. Seine zwei Buchveröffentlichungen zum Thema: "Krise und Zukunft des Sozialstaates" sowie "Kinderarmut in Ost- und Westdeutschland", Wiesbaden (VS-Verlag für Sozialwissenschaften) 2005 nuität prägte die SPD maßgeblich Programm und Politik der von einer Unionspolitikerin geführten Bundesregierung.

\section{Die SPD zwischen Zukunftsgestaltung und der Beschäftigung mit sich selbst}

Die tiefe Krise der SPD schlug sich nicht zuletzt in einem raschen Verschleiß ihrer Parteivorsitzenden auf Bundesebene nieder. Während die Koalitionsverhandlungen mit der Union das entscheidende Stadium erreichten, trat Franz Müntefering, der Oskar Lafontaine wegen dessen überstürzten Abgangs immer heftig kritisiert hatte, selbst aus einem viel nichtigeren Anlass (Nominierung der Parteilinken Andrea Nahles für die Position einer Generalsekretärin) zurück.

Der brandenburgische Ministerpräsident Matthias Platzeck, auf dem Karlsruher Parteitag am 15. November 2005 mit einem Rekordergebnis zum SPD-Vorsitzenden gewählt, amtierte glücklos und trat bereits am 10. April 2006 zurück. Am selben Tag erschien im Spiegel ein Artikel von Platzeck, der sein Leitbild des „vorsorgenden Sozialstaates“ erläuterte: „Der vorsorgende Sozialstaat für das 21. Jahrhundert investiert in die Menschen und ihre Fähigkeiten. Er fördert Beschäftigung, setzt auf Gesundheitsprävention und verhindert Armut. Er gestaltet den demografischen Wandel mit den Betroffenen, und er erkennt die existentielle Bedeutung von Bildung für die einzelnen Menschen wie auch für die Zukunft unserer Gesellschaft an. Er ist Partner, nicht Verwalter der Menschen. Er macht Angebote, um ihre Stärken zu entwickeln. Er aktiviert die Menschen, damit sie ihr Leben in eigener Verantwortung gestalten können. Der vorsorgende Sozialstaat ist nicht Wachstumshindernis, sondern wirtschaftliche Produktivkraft; er muss dafür anders, we- 
niger als bislang durch Sozialversicherungsbeiträge finanziert werden."3

Nach der „Ära Schröder“ orientierte sich die SPD nicht mehr an „New Labour“, sondern eher am skandinavischen Wohlfahrtsmodell, welches aus ihrer Sicht vornehmlich beinhaltet: „Investitionen in Bildung und Betreuung, flexicurity am Arbeitsmarkt, Grundsicherung und steuerfinanzierte soziale Sicherungssysteme. ${ }^{\text {" }}$ Bei dem zuletzt genannten Strukturelement, das immer stärker in den Blickpunkt der Regierungspolitik rückt, handelt es sich jedoch um eine willkürliche, auf das hierzulande übermächtige neoliberale Dogma einer Senkung der Lohnnebenkosten bezogene Interpretation, speist sich die Sozialversicherung im „schwedischen Volksheim“ doch keineswegs nur aus Steuermitteln, sondern auch aus Versicherungsbeiträgen, welche - abgesehen von der Altersvorsorge, wo sich die Arbeitnehmer/innen seit einiger Zeit beteiligen - sogar ausschließlich die Arbeitgeber entrichten.

Platzecks Nachfolger wurde der rheinland-pfälzische Ministerpräsident Kurt Beck, der die von Helmut Kohl beim Regierungsantritt 1982 gewählte Parole „Leistung muss sich wieder lohnen!“ aufgriff, als er in der Welt am Sonntag vom 20. August 2006 die Befürchtung äußerte, dass der Leistungsgedanke durch aktuelle Entwicklungsprozesse der Finanzmärkte und der Arbeitswelt untergraben werde: „Die politisch wichtige Frage ist, wie lange eine Gesellschaft europäischer Tradition es ohne Schaden aushält, wenn ein Kernversprechen der sozialen Marktwirtschaft, Sicherheit und Aufstieg durch Arbeitsleistung zu bieten, immer wieder gebrochen wird. " ${ }^{5}$ Fragen der Leistungsgerechtigkeit werfe die Finanzierung der sozialen Sicherheit in Deutschland dadurch auf, dass sich immer mehr Arbeitsverhältnisse dem Solidarprinzip entzögen. Nur aus sozialversicherungspflichtigen Beschäftigungsverhältnissen würden Beiträge in die Versicherungskassen geleistet, und längst müssten beträchtliche Summen aus Steuern zugeschossen werden: „Abhängig Beschäftigte zahlen heute zweimal: als Steuerpflichtige und als Beitragszahler. Das vergrößert nicht nur den Abstand zwischen Brutto- und Nettolohn, es erhöht mit den Arbeitskosten auch das Arbeitsplatzrisiko. Deshalb ist ein Wechsel erforderlich, der Lohnnebenkosten senkt und die sozialen Sicherungssysteme stärker durch Steuern finanziert. "6 Die zur Gewährleistung des sozialen Zusammenhalts unseres Gemeinwesens nötigen Aufwendungen dürften nicht nur einer Gruppe aufgebürdet, vielmehr müssten die dadurch entstehenden Kosten von allen nach Maßgabe ihrer Möglichkeiten getragen werden.

Kurz darauf verlangte Beck im stern eine stärkere Hinwendung der Sozialdemokraten zu den von Gerhard Schröder zunächst als „Neue Mitte“ bezeichneten Bevölkerungsschichten: „Die SPD soll wieder Hoffnungsträger für viele Menschen werden - nicht nur für diejenigen, die Unterstützung brauchen, sondern für alle, die an einer gerechten, forschungsorientierten und technologiefreundlichen Gesellschaft interessiert sind. " Versuch einer Öffnung und Verbreiterung der Wähler- basis klang, bedeutete im Grunde, dass die SPD ihren Anspruch, eine Volkspartei zu sein, fallen und die von den Sozialreformen gebeutelten Unterschichten, die Beck nur noch in einem Nebensatz erwähnte, kampflos der Linkspartei.PDS überließ. So erklärte Beck, dass es nicht machbar sei, einen allgemeinen Mindestlohn gesetzlich festzulegen, obwohl Vizekanzler Müntefering lange diesbezügliche Hoffnungen geweckt hatte, sei es, um den Gewerkschaften, die darüber zunächst uneins waren, ein „Versöhnungsangebot“ zu machen oder sie auseinanderzudividieren. Gleichheit als ursprüngliches Ziel der SPD gab Beck praktisch auf: „Mir geht es um soziale Gerechtigkeit. Es muss Verteilungsgerechtigkeit geben, aber sie ist nicht der Maßstab aller Dinge. Chancengerechtigkeit ist mindestens genauso wichtig. "8

Hier knüpfte Beck an die Ideen von Peer Steinbrück, seinem Stellvertreter im Parteivorsitz, an. In einer Grundsatzrede beim Neujahrsempfang der Industrie- und Handelskammer Frankfurt/Main am 10. Januar 2006 hatte der neue Bundesfinanzminister die „Chancengerechtigkeit" statt der Ergebnisgleichheit zum Grundprinzip eines modernen, die Bürger „aktivierenden“ und ihre „übertriebene Anspruchshaltung“ bekämpfenden Sozialstaates erklärt. Dessen alleiniges Ziel könne es, sagte Steinbrück weiter, nicht sein, „jeden Einzelnen gegen alle Unwägbarkeiten des Marktes zu schützen. Das erste Ziel muss mehr denn je werden, den Einzelnen zur Teilnahme und Teilhabe auf den Märkten zu befähigen. Die Schlüssel dazu sind Bildung und Qualifizierung in ihrer ganzen Palette. ${ }^{9}$ Wenn die SPD den künftigen als „vorsorgenden Sozialstaat" bezeichnet, schwingt die Abwertung des bisherigen als eines „nachsorgenden Sozialstaates" mit. Welcher politisch-ideologische Sprengstoff in diesem Begriffspaar steckt, brachte der frühere Wirtschafts- und Arbeitsminister Clement zum Ausdruck, als er in der Welt am Sonntag schrieb: „Heute geht es um den Abschied vom Wohlfahrtsstaat und die Hinwendung zum sozialen Bildungsstaat. (...) Der Sozialstaat in seiner bisherigen Ausprägung und Ausstattung trägt nicht mehr, und wir können ihn auch nicht mehr finanzieren. Wir müssen ihn deshalb nicht bloß umbauen, er braucht ein neues Fundament, eine neue Statik."10

\section{Weder genug Mut noch Menschlichkeit - der Koalitionsvertrag und die Regierungserklärung}

Die am 11. November 2005 ausgehandelte und eine Woche später von den Spitzenrepräsentanten der CDU, CSU und SPD unterzeichnete Koalitionsvereinbarung war von finanz- wie sozialpolitischer Buchhaltermentalität geprägt, wohingegen jedes Signal für einen Neuanfang, visionäre Konzepte und Alternativen zum rot-grünen Regierungskurs fehlten. Schon die Überschrift „Gemeinsam für Deutschland - mit Mut und Menschlichkeit“ ließ deutlich erkennen, dass die Koalitionäre im Wesentlichen nach Kontinuität strebten, selbst politische Plattitüden und nationales Pathos jedoch nicht scheuten, wenn es darum ging, ihre Politik zu legitimieren. 


\section{ZUR DISKUSSION GESTELLT}

Bereits im Einleitungssatz der Präambel findet man mit der Globalisierung und dem demografischen Wandel die beiden Großen Erzählungen unserer Zeit, verbunden mit der Absicht, weitere Einschnitte in das System der sozialen Sicherheit als Sachzwang und nicht als Resultat eigener Überzeugungen und programmatisches Ziel erscheinen zu lassen: „Deutschland steht vor großen Herausforderungen: Arbeitslosigkeit, Staatsverschuldung, demografischer Wandel und der Veränderungsdruck der Globalisierung verlangen große politische Anstrengungen, um heutigen und künftigen Generationen ein Leben in Wohlstand zu sichern."11 CDU, CSU und SPD erklärten weiter, das Vertrauen in die Funktionsfähigkeit der Gesetzlichen Renten-, Pflege- und Krankenversicherung durch ihre Regierungspolitik stärken zu wollen: „Wir werden die notwendigen Reformschritte unternehmen, um auch heute und morgen soziale Sicherheit zu gewährleisten. Dazu gehört, dass die Lasten zwischen Jung und Alt gerecht verteilt werden, dass mit der gestiegenen Lebenserwartung das Renteneintrittsalter ansteigt und die private Altersvorsorge vor allem der jungen Familien besser gefördert wird. Die elementaren Lebensrisiken Krankheit, Alter, Pflegebedürftigkeit, Arbeitslosigkeit bleiben solidarisch abgesichert."12

Am 30. November 2005 gab Angela Merkel im Bundestag ihre Regierungserklärung ab. Darin nannte sie als Ziel, die Rahmenbedingungen dafür zu schaffen, dass Deutschland in 10 Jahren „wieder unter den ersten Drei in Europa“ zu finden sei: „Wir wollen den Föderalismus neu ordnen, wir wollen den Arbeitsmarkt fit machen, wir wollen unsere Schulen und Hochschulen wieder an die Spitze führen, wir wollen unsere Verschuldung bändigen und unser Gesundheits- und Rentensystem und die Pflege in Ordnung bringen. "13 Ein berühmtes Wort von Willy Brandt abwandelnd, das jenseits der Mauer wie Musik geklungen habe, sagte Merkel: „Lasst uns mehr Freiheit wagen!“ und fügte erläuternd hinzu: „Lassen Sie uns die Wachstumsbremsen lösen! Lassen Sie uns selbst befreien von Bürokratie und altbackenen Verordnungen. "14 Merkel erklärte, gleichermaßen ein Herz für die Schwachen (Kranke, Kinder, Alte) wie für Leistung zu haben, und versprach, bei der geplanten Überarbeitung von Hartz IV für „mehr Gerechtigkeit und weniger Missbrauch“ zu sorgen.

Die neue Regierung folgte der alten Philosophie, wonach die Arbeitslosigkeit sinkt, wenn man mit den Beiträgen (der Arbeitgeber) zur Sozialversicherung die gesetzlichen Lohnnebenkosten drückt. Es kommt aber gar nicht auf deren, vielmehr auf die Höhe der Lohnstückkosten an, welche in der Bundesrepublik wegen einer überproportional wachsenden Arbeitsproduktivität seit Jahren weniger stark steigen als in den mit ihr konkurrierenden Ländern und zuletzt sogar sanken, was $2005 \mathrm{zu}$ einem Rekordexportüberschuss in Höhe von 160,5 Mrd. EUR führte.

\section{Hauptleidtragende der Großen Koalition}

Die kleinen Leute gehören zu den Verlierern der Großen Koalition; Hauptleidtragende etwa der Mehrwertsteuererhöhung dürften Familien von Geringverdiener(inne)n, (Langzeit-)Arbeitslose, Sozialhilfebezieher/innen, Studierende, Schüler/innen und Rentner/innen sein. Gleich zu Beginn machte die Große Koalition deutlich, dass mit Rentenerhöhungen vorläufig nicht zu rechnen sei, sondern in den nächsten Jahren mehrere „Nullrunden“ anstünden. Rentenkürzungen schloss der Koalitionsvertrag zwar für die ganze Legislaturperiode aus, er sah aber zwecks Gewährleistung der Beitragssatzstabilität die Möglichkeit, „nicht realisierte Dämpfungen von Rentenanpassungen nachzuholen“, sowie die „schrittweise, langfristige Anhebung des gesetzlichen Renteneintrittsalters" vor. ${ }^{15}$

Während mit einem „Nachholfaktor“ im Rentenrecht erreicht werden soll, dass Kürzungen, auf die zunächst verzichtet wurde, in Erhöhungsphasen letztlich doch noch - weniger spektakulär - wirksam werden, wollten CDU, CSU und SPD die Lebensarbeitszeit unter Hinweis auf den demografischen Wandel verlängern und 2007 die gesetzliche Grundlage für eine 2012 beginnende und für den ersten Jahrgang bis spätestens 2035 abgeschlossene Anhebung der Regelaltersgrenze von 65 auf 67 Jahre schaffen. Völlig unerwartet preschte Arbeits- und Sozialminister Müntefering im Januar 2006 mit der auch seine eigene Partei überraschenden und viele Genossen, die sich damals gerade in Landtagswahlkämpfen befanden, verärgernden Idee vor, das gesetzliche Renteneintrittsalter schneller anzuheben, als es die sog. Rürup-Kommission empfohlen und der Koalitionsvertrag festgeschrieben hatte: Nach dem am 1. Februar 2006 auf Drängen des Vizekanzlers vom Bundeskabinett gefassten Beschluss erhöht sich das Regelrentenalter im Jahr 2012 für den Geburtsjahrgang 1947 um einen und für Folgejahrgänge jedes Jahr um einen weiteren Monat, bis der Jahrgang 1958 im Alter von 66 eine abschlagsfreie Rente ab 2023 bezieht; für die Folgejahrgänge beschleunigt sich die Anhebung der Altersgrenze um jeweils 2 Monate pro Jahr, bis der Jahrgang 1964 bereits 2029 erst mit 67 in Rente gehen kann. Angesichts der Tatsache, dass die meisten deutschen Unternehmen gar keine Arbeitnehmer/innen über 50 beschäftigen, führt diese „Reform“ zu weiteren Rentenkürzungen, zwingt sie doch noch mehr Beschäftigte, vor Erreichen der Regelaltersgrenze - und das heißt: mit entsprechenden Abschlägen - in den Ruhestand zu gehen.

Da half es wenig, dass Müntefering mit einer „Initiative 50plus" zur beruflichen Wiedereingliederung älterer Langzeitarbeitsloser (Zahlung von Kombilöhnen und Lohnzuschüssen an die Arbeitgeber), zur Weiterbildung älterer Arbeitnehmer/innen (Ausgabe von Bildungsgutscheinen) sowie zur Gestaltung altersgerechter Arbeitsbedingungen („Beschäftigungspakte“) die Chancen dieser Bevölkerungsgruppe verbessern wollte. Für den Tübinger Hochschullehrer Josef Schmid bildet die Verlängerung der Lebensarbeitszeit neben der „einnahmenorientierten 
Ausgabepolitik“ durch die Riester-Reform den „zweiten Paradigmawechsel in der Rentenpolitik“, welcher diese stärker als Qualifizierungs- und Beschäftigungspolitik denn als reine (transferorientierte) Sozialpolitik konturiere, aber „erhebliche Akzeptanzprobleme“ mit sich bringe und „enorm hohe Anforderungen an die politische Steuerung und Konsensfindung" stelle. ${ }^{16}$

Die von der Großen Koalition im Ersten SGB-II-Änderungsgesetz vom 22. Dezember 2005, im Zweiten SGB-IIÄnderungsgesetz vom 24. März 2006 und im Hartz-IV-Fortentwicklungsgesetz vom 25. Juli 2006, das zuerst großspurig „Optimierungsgesetz" heißen sollte, enthaltenen „Korrekturen“ an der rot-grünen Arbeitsmarktreform liefen trotz einzelner Verbesserungen für die Langzeitarbeitslosen (Anhebung des Regelsatzes in Ostdeutschland von 331 auf 345 EUR wie in Westdeutschland) größtenteils auf eine Kürzung des Leistungsumfangs sowie eine Verschärfung der Kontrollmaßnahmen hinaus. Damit wollte man Leistungsmissbrauch aufdecken und die Kosten senken. Die beschlossenen Regelungen, mit denen jährlich mehrere Mrd. EUR eingespart werden sollen, kann man durchaus als „Hartz V“ bezeichnen, stellen sie doch eine Fortsetzung und massive Verschärfung des Drucks auf Arbeitsuchende dar.

Betroffen waren vor allem Heranwachsende und junge Erwachsene, die von zu Hause auszogen und als Arbeitsuchende mittels der Grundsicherung nach dem SGB II lieber eine eigene Bedarfsgemeinschaft gründeten, als im Haushalt ihrer Eltern zu verbleiben. Mit dem Zweiten SGBII-Änderungsgesetz wurden Kinder, die das 25. Lebensjahr noch nicht vollendet haben, zur Bedarfsgemeinschaft ihrer Eltern gerechnet und der Regelsatzbedarf für sie vom 1. April 2006 an auf 80 Prozent reduziert. Damit ging man weit über zivilrechtliche Unterhaltsverpflichtungen der Eltern für ihre erwachsenen Kinder hinaus: „Gerade noch verhindert werden konnte die Einstandspflicht des Stiefelternteils für Kinder des Partners in einer Lebensgemeinschaft. "17 Wenn die jungen Menschen einen eigenen Hausstand gründen wollen, müssen sie nunmehr vorher die Zustimmung des kommunalen Leistungsträgers einholen. Ziehen sie ohne dessen Einwilligung bei ihren Eltern aus, erhalten sie bis zur Vollendung des 25. Lebensjahres gleichfalls nur 80 Prozent der Regelleistung. Heranwachsende und junge Erwachsene wieder in der Abhängigkeit von ihren Eltern zu belassen und ihnen per Mittelentzug die Möglichkeit der Gründung eines eigenen Hausstandes zu nehmen, ist einer so wohlhabenden und hoch individualisierten Gesellschaft, die im Zeichen der Globalisierung berufliche Flexibilität und geografische Mobilität von ihren Mitgliedern verlangt, unwürdig.

Mit dem Gesetz zur Fortentwicklung der Grundsicherung für Arbeitsuchende wurde die Beweislast bei eheähnlichen Gemeinschaften umgekehrt: Musste vorher der Leistungsträger nachweisen, dass eine solche bestand, wenn der Antragsteller und eine weitere Person länger als 1 Jahr in einer Wohnung zusammen lebten, muss dieser seit dem 1. August 2006 im Zweifelsfall den Verdacht widerlegen, dass es sich bei ihm und dem Mitbewohner bzw. der Mitbewohnerin um eine Bedarfsgemeinschaft handelt. Flächendeckend soll ein Außendienst prüfen, ob die Anspruchsvoraussetzungen gegeben sind, und Missbrauch vorbeugen oder begegnen. Zum selben Zweck kann die Agentur für Arbeit künftig Daten aus dem Kraftfahrzeugbundesamt, dem Melderegister und dem Ausländerzentralregister abrufen.

Gleichzeitig wurde die Höhe der Vermögensfreibeträge verändert: Während der Freibetrag für die Altersvorsorge von 200 auf 250 EUR pro Lebensjahr stieg, sank der Grundfreibetrag von 200 auf 150 EUR pro Lebensjahr bei entsprechender Anpassung der Höchstgrenzen, woraus Einsparungen für den Bund resultieren dürften, weil nur relativ geringe Sparsummen einer Zweckbindung zugunsten der Alterssicherung unterliegen. Erstantragsteller(inne)n soll künftig sofort ein Job oder eine Fortbildung angeboten werden, um ihre Arbeitswilligkeit zu testen. Lehnt ein Antragsteller das Angebot ab oder verletzt er drei Mal seine Mitwirkungspflicht während eines Jahres, droht ihm ab 1. Januar 2007 ein vollständiger Leistungsentzug. Bei Jugendlichen unter 25 Jahren erstreckt sich diese Sanktion im Fall einer wiederholten Pflichtverletzung sogar auf die Kosten von Unterkunft und Heizung. Während sich dadurch die Jugendarmut erhöhen dürfte, trägt eine andere Neuregelung zur Vermehrung der materiellen Not älterer Menschen bei: Nach dem 31. Dezember 2006 sinkt der für Alg-II-Empfänger/innen abgeführte Rentenversicherungsbeitrag von 78 auf 40 EUR pro Monat, wodurch sich die Leistungsansprüche im Alter weiter verringern.

Um das durch die Hartz-Gesetze erleichterte Lohndumping einzudämmen, wurde der Geltungsbereich des bisher vor allem in der Bauindustrie wirksamen Arbeitnehmer-Entsendegesetzes vom 26. Februar 1996 auf die Gebäudereinigungsbranche ausgedehnt. Dagegen lehnten CDU und CSU die Einbeziehung des perspektivisch weitaus wichtigeren Zeitarbeitssektors ab. Vermutlich spielte hierbei die Tatsache eine Rolle, dass der höhere Tarifabschluss des DGB und nicht der geringere des Christlichen Gewerkschaftsbundes in diesem Bereich für allgemein verbindlich erklärt worden wäre. ${ }^{18}$ Aber selbst Arbeitsund Sozialminister Müntefering, der die Debatte angestoßen hatte, rang sich nicht zur Einführung eines allgemeinen gesetzlichen Mindestlohnes durch, den es in einer Mehrheit der EU-Mitgliedstaaten längst gibt.

CDU, CSU und SPD haben im Koalitionsabkommen vom 11. November 2005 vereinbart, den Kündigungsschutz „weiterzuentwickeln“ und auf diesem Gebiet größere Transparenz und Rechtssicherheit sowohl für Beschäftigte wie auch für Arbeitgeber zu schaffen: „Wir werden daher auf der einen Seite die Möglichkeit streichen, Arbeitsverträge in den ersten 24 Monaten für denselben Zeitraum sachgrundlos zu befristen. Gleichzeitig geben wir den Arbeitgebern bei der Neueinstellung die Option an die Hand, anstelle der gesetzlichen Regelwartezeit von 6 Monaten bei der Begründung des Arbeitsverhältnisses mit dem Einzustellenden eine Wartezeit von 


\section{ZUR DISKUSSION GESTELLT}

bis zu 24 Monaten zu vereinbaren."19 Der gesetzliche Kündigungsschutz sollte gelockert und die Probezeit, in der er nicht greift, von 6 Monaten auf 2 Jahre verlängert werden. Dafür entfiele im Gegenzug die Möglichkeit der Unternehmen, Arbeitsverhältnisse ohne sachlichen Grund zu befristen.

Die französische Nationalversammlung verabschiedete wenige Wochen später, im Februar 2006, ein neues Arbeitsgesetz, das mittels des „Vertrages zur Ersteinstellung" (Contrat Première Embauche, CPE) für Arbeitnehmer/innen unter 26 Jahren in Unternehmen mit mehr als 20 Beschäftigten den Kündigungsschutz für 2 Jahre aufhob. Dagegen demonstrierten nicht nur Hunderttausende junger Leute, vor allem Schüler/innen und Studierende, sondern auch die Richtungsgewerkschaften und Kulturschaffende in einem breiten Bündnis. Aufgrund der massenhaften Proteste, die bis zu Universitätsbesetzungen und Straßenblockaden gingen, zog die bürgerliche Regierung von Premierminister Dominique de Villepin das Gesetz am 7. April 2006 zurück. Man darf vermuten, dass die Große Koalition ihren Plan, der dieser Regelung bis aufs Haar gleicht, aber nicht nur die Entrechtung jüngerer Arbeitnehmer/innen vorsieht, auch deshalb sehr zögerlich verfolgt hat, zumal selbst die Wirtschaftsverbände wenig Sympathien dafür erkennen ließen.

\section{Zusammenfassung und Ausblick}

Dass die Vorschusslorbeeren, mit denen man Angela Merkel bei ihrem Amtsantritt als Bundeskanzlerin und nach ihren ersten öffentlichen Auftritten vor allem auf diplomatischem Parkett bedachte, schnell welkten und die Große Koalition ihren Kredit bei einer überwiegenden Bevölkerungsmehrheit geradezu im Rekordtempo verspielt hat, dürfte mit der Art und Weise zu tun haben, wie sie den rot-grünen Reformkurs im Sozialbereich noch verschärfte und die Umverteilung von unten nach oben fortsetzte.

Die schwarz-roten Reformen sind von zahlreichen Brüchen und Widersprüchen gekennzeichnet. Weder lässt sich in der Sozialpolitik bisher eine klare Linie erkennen, noch wird sie konsequent durchgehalten. Da sucht man mit den Sozialversicherungsbeiträgen die Lohnnebenkosten zu senken, kompensiert aber die Senkung der Beiträge zur Arbeitslosenversicherung zum 1. Januar 2007 durch eine nur unwesentlich geringere Erhöhung der Rentenversicherungs- und Krankenkassenbeiträge. Man will „versicherungsfremde Leistungen“ wie die beitragsfreie Mitversicherung der Kinder stärker über Steuern finanzieren, streicht den Krankenkassen aber gleichzeitig bisher gewährte Zuschüsse aus der Tabaksteuer und erhöht ihre laufenden Kosten durch die Anhebung der Mehrwertsteuer. Vizekanzler Müntefering, im Bermudadreieck zwischen Mindestlöhnen, Kombilöhnen und Zuverdienstgrenzen beim Alg II steckend, dachte laut über deren Senkung nach, um die große Zahl der aufstockenden Geringverdiener/innen einzudäm- men, obwohl die damals noch von ihm selbst geführte Mehrheitsfraktion des Bundestages die entsprechenden Freibeträge erst zum 1. Oktober 2005 angehoben hatte, um Langzeitarbeitslosen größere finanzielle Anreize zur Arbeitsaufnahme zu geben, usw.

Insgesamt erscheint die schwarz-rote Reformpolitik weniger spektakulär als die mit dem Namen von Peter Hartz verbundene Arbeitsmarktreform, wirkt subtiler als diese und hat manchmal auch stärker Stückwerkcharakter, sie ist aber keineswegs sozial gerechter. Denn die CDU/CSU/SPD-Regierung verteilt großzügige Steuergeschenke an die „oberen Zehntausend“, während sie vor allem gegenüber den Unterschichten zugeknöpft und kleinkariert agiert. Wohin ein Kurs der Spaltung in Gewinner und Verlierer/innen zusammen mit einer ausgeprägten sozialräumlichen Segregation (Gettoisierung) führen kann, haben die oft als "Jugendkrawalle“ missdeuteten Gewaltakte marginalisierter Bewohner der französischen Banlieues im Oktober/November 2005 gezeigt.

Hierzulande entstand zur selben Zeit eine Regierungskoalition gegen den Sozialstaat, der nicht nur für die Massenarbeitslosigkeit verantwortlich, sondern auch zum Sündenbock einer falschen Wirtschafts-, Steuer- und Finanzpolitik der etablierten Parteien gemacht wird. Nach neoliberaler Lesart erhöht die Freiheit von Wirtschaftssubjekten, die unternehmerisch tätig sind, automatisch den gesellschaftlichen Wohlstand. Für leistungsunfähige bzw. -unwillige Mitglieder der Gesellschaft bringt sie eine größere Marktabhängigkeit und geringere Existenzsicherheit mit sich. Weniger Sozialstaat bedeutet allerdings mitnichten mehr Freiheit, sondern größere Ungleichheit, mehr soziale Ungerechtigkeit und wachsende Unzufriedenheit in der Bevölkerung.

Legitimiert durch den in Fach- und Medienöffentlichkeit ständig wiederholten Hinweis auf Sachzwänge haushaltspolitischer Art bzw. tiefgreifende gesellschaftliche Wandlungsprozesse (Globalisierung sowie „Vergreisung" und „Schrumpfung“ der Bevölkerung durch die demografische Entwicklung) einerseits sowie eine sukzessive Umdeutung des Gerechtigkeitsbegriffs (von der Bedarfszur „Leistungsgerechtigkeit“, der Verteilungs- zur „Teilhabegerechtigkeit“ und der sozialen zur „Generationengerechtigkeit") andererseits, wird der Wohlfahrtsstaat mittels verharmlosend und beschönigend „Reformen" genannter Maßnahmen der Großen wie der schwarz-gelben und der rot-grünen Koalition vor ihr nicht nur systematisch demontiert, sondern auch in mehrfacher Hinsicht transformiert:

1. Aus dem Wohlfahrtsstaat wird ein „nationaler Wettbewerbsstaat" (Joachim Hirsch), der die Aufgabe hat, durch seine Politik die Konkurrenzfähigkeit des „eigenen" Wirtschaftsstandortes auf dem Weltmarkt, Wachstum und Beschäftigung zu fördern. Sozialstaatlichkeit, die eigentlich Verfassungsrang hat, besitzt für Neoliberale keinen Eigenwert mehr, sondern muss sich nach der Standortlogik wirtschaftlichen und Machtinteressen unterwerfen. Dies zeigt sich etwa bei Debatten über die Lockerung des Kündigungsschutzes oder die 
Aufweichung des Flächentarifvertrages. Da fast alle Gesellschaftsbereiche im Zuge einer Ökonomisierung, Privatisierung und Liberalisierung nach dem Vorbild des Marktes umstrukturiert werden, hält die Konkurrenz auch Einzug im Sozialstaat.

\section{Aus dem Sozialstaat wird ein Minimalstaat. Der} „schlanke Staat“, wie er dem Neoliberalismus vorschwebt, ist im Hinblick auf die Sozialpolitik eher magersüchtig, aber keineswegs frei von bürokratischen Auswüchsen - ganz im Gegenteil! Leistungskürzungen und die Verschärfung von Anspruchsvoraussetzungen gehen mit Strukturveränderungen einher, die nicht nur mehr Markt, sondern teilweise auch mehr staatliche Administration bedeuten. Beispielsweise werden für Zertifizierungsagenturen, Evaluationsbürokratien und Leistungskontrollen aller Art womöglich mehr Sach- und Personalmittel benötigt als vorher.

3. Der neoliberale Residualstaat ist eher Kriminal- als Sozialstaat, weil ihn die drastische Reduktion der Wohlfahrt zur Repression gegenüber jenen Personengruppen zwingt, die als Modernisierungs- bzw. Globalisierungsverlierer/innen zu Opfern seiner rückwärts gerichteten „Reformpolitik“ werden. Je weniger großzügig die Sozialleistungen einer reichen Gesellschaft ausfallen, umso schlagkräftiger muss ihr Sicherheitsbzw. Gewaltapparat sein. Was sie an der Wohlfahrt spart, geht für Maßnahmen gegen den Drogenmissbrauch, Kriminalität und Gewalt drauf.

4. An die Stelle des aktiven Sozialstaates, wie man ihn bei uns bisher kannte, tritt - sehr stark vom Kommunitarismus, einer US-amerikanischen Denkrichtung, beeinflusst - ein „aktivierender", Hilfebedürftige nicht mehr ohne entsprechende Gegenleistung alimentierender Sozialstaat. Der „welfare state“ wandelt sich zum „workfare state“, wenn man den Arbeitszwang ins Zentrum der Beschäftigungs- und Sozialpolitik rückt. Ausgerechnet in einer schweren Beschäftigungskrise, wo Millionen Arbeitsplätze - nicht: Arbeitswillige - fehlen, wird so getan, als seien die von Erwerbslosigkeit unmittelbar Betroffenen an ihrem Schicksal selbst schuld. Trotz des wohlklingenden Mottos „Fördern und fordern!“, das Leistungszusagen von Gegenleistungen der Begünstigten abhängig macht, bemüht man sich gar nicht mehr ernsthaft darum, die Chancen von sozial Benachteiligten zu verbessern, wie die Tatsache zeigt, dass im Weiterbildungsbereich immer stärker kurze Trainingsmaßnahmen dominieren.

5. Der deutsche Sozial(versicherungs)staat, seit seiner Begründung durch Otto von Bismarck im Kern darauf gerichtet, die männlichen Industriearbeiter mit ihren Familien vor Standardrisiken wie dem Tod des Ernährers, der Invalidität und der Armut im Alter zu schützen, wird zu einem Fürsorge-, Almosen- und Suppenküchenstaat gemacht, der nicht mehr den Lebensstandard seiner Klientel erhält, sondern ihr nur noch eine Basisversorgung angedeihen lässt. Hartz IV war mit seiner Abschaffung der Arbeitslosenhilfe, als „Zusammenlegung mit der Sozialhilfe" sehr unzureichend charak- terisiert, ein wichtiger Zwischenschritt auf diesem Weg und eine historische Zäsur in der Entwicklung des Arbeits- und Sozialrechts. Man spricht von „Eigenverantwortung", "Selbstvorsorge" und "Privatinitiative", meint aber die öffentliche Verantwortungslosigkeit sowie eine Mehrbelastung von Arbeitnehmer(inne)n und Rentner(inne)n.

6. Gleichzeitig wird das Gemeinwesen in einen Wohlfahrtsmarkt und einen Wohltätigkeitsstaat gespalten. Auf dem Wohlfahrtsmarkt kaufen sich jene Bürger/innen, die es sich finanziell leisten können, das für sie erschwingliche Maß an sozialer Sicherheit (z.B. Altersvorsorge durch Versicherungspolicen der Assekuranz). Ergänzend stellt der postmoderne Fürsorgestaat als "Grundsicherung" bezeichnete Minimalleistungen bereit, die Menschen vor dem Verhungern und Erfrieren bewahren, sie jedoch der Privatwohltätigkeit überantworten. Folgerichtig haben karitatives Engagement, ehrenamtliche Tätigkeit im Sozial- und Gesundheitsbereich, Spendenfreudigkeit und die Gründung gemeinnütziger Stiftungen (wieder) Hochkonjunktur.

7. Die sozialpolitische Postmoderne trägt beinahe mittelalterliche Züge und lässt einen Rückfall in den Feudalismus befürchten. Durch die schrittweise Reindividualisierung, Reprivatisierung und Rückverlagerung sozialer Risiken auf die Familien, wie sie CDU-Generalsekretär Ronald Pofalla mit seinem Vorschlag untermauerte, im Falle der Arbeitslosigkeit nicht nur die Eltern für ihre erwachsenen Kinder, sondern auch die volljährigen Kinder für ihre Eltern zahlen zu lassen, lässt man Errungenschaften des gesellschaftlichen Modernisierungsprozesses hinter sich.

Stattdessen müsste der Sozialstaat an die jüngsten Entwicklungen in der Arbeitswelt (Tendenz zur Prekarität von Beschäftigungsverhältnissen) und im familiären Zusammenleben der Menschen (verstärkter Hang zur Individualität) angepasst werden. Nötig wäre eine Kurskorrektur, die wieder für mehr Gerechtigkeit sorgen und eine gleichmäßigere Verteilung des gesellschaftlichen Reichtums zum Ziel haben müsste.

Wenn die Regierung Merkel/Müntefering scheitert und die Massenarbeitslosigkeit nicht dauerhaft sinkt, wird der Ruf nach dem „starken Mann“ lauter als bisher erschallen, vor allem mit der Begründung, dass die erste Frau im Kanzleramt der Probleme nicht Herr geworden sei, aber vermutlich auch mit der Konnotation, dass nunmehr die Zügel straffer angezogen und autoritäre Herrschaftsmethoden praktiziert werden müssten, um die Lage in den Griff zu bekommen. Mit der schwarz-gelben, der rot-grünen und der schwarz-roten Koalition sind fast alle Farbkombinationen im Regierungsalltag „erprobt“ worden, was die Gefahr erhöht, dass sich rechtsextreme bzw. -populistische Tendenzen verstärken. Demagogen könnten als „Retter des Sozialstaates“ in Erscheinung treten und Gewinner wie Verlierer/innen seines Um- bzw. Abbaus gleichermaßen bei ihnen Zuflucht suchen. 


\title{
ZUR DISKUSSION GESTELLT
}

\section{Fußnoten}

1 Vgl. kritisch hierzu: Christoph Butterwegge, Krise und Zukunft des Sozialstaates, 3. Aufl. Wiesbaden 2006, S. $184 \mathrm{ff}$.

2 Rudolf Walther, Ohne Tradition keine politische Zukunft. Die drei strategischen Optionen der SPD, in: Neue Gesellschaft/Frankfurter Hefte 9/2005, S. 27

3 Matthias Platzeck, Ein besserer Sozialstaat, in: Der Spiegel v. 10.4.2006, S. 35

4 Stefan Grönebaum, „Innovation statt Gerechtigkeit“ wäre die falsche Dialektik. Replik auf Tobias Dürr und Manfred Güllner (in: NG/FH 78/2005), in: Neue Gesellschaft/Frankfurter Hefte 9/2005, S. 46 (Hervorh. im Original, Ch.B.)

5 Kurt Beck, Leistung muss sich wieder lohnen, in: Welt am Sonntag v. 20.8.2006

6 Ebd.

7 „35 Prozent plus ein dickes X“. Ab in die Mitte! Politik für die Leistungsträger! SPD-Chef Kurt Beck verkündet im stern-Gespräch einen Kurswechsel seiner Partei - und sein Wahlziel für 2009, in: stern v. 31.8 .2006$, S. 60

8 Ebd., S. 58

9 Peer Steinbrück, Reduzierung auf die Kernaufgaben. Rede des Bundesministers der Finanzen beim Neujahrsempfang der Industrie- und Handelskammer Frankfurt am Main am 10. Januar 2006, in: Blätter für deutsche und internationale Politik 2/2006, S. 249
10 Wolfgang Clement, Ein neues „Godesberg“ für die SPD, in: Welt am Sonntag v. 14.5.2006

11 CDU Deutschlands/CSU Landesleitung/SPD Deutschlands (Hrsg.), Gemeinsam für Deutschland. Mit Mut und Menschlichkeit. Koalitionsvertrag von CDU, CSU und SPD, Rheinbach o.J., S. 15

12 Ebd., S. 16

13 Mehr Freiheit wagen. Auszüge aus der ersten Regierungserklärung von Bundeskanzlerin Angela Merkel vor dem Deutschen Bundestag, in: Frankfurter Rundschau v. 1.12.2005

14 Ebd.

15 Siehe CDU Deutschlands/CSU Landesleitung/SPD Deutschlands (Hrsg.), Gemeinsam für Deutschland, a.a.O., S. 96

16 Siehe Josef Schmid, Rentenreform: Überlebt der Wohlfahrtsstaat? in: Roland Sturm/Heinrich Pehle (Hrsg.), Wege aus der Krise? - Die Agenda der zweiten Großen Koalition, Opladen/Farmington Hills 2006, S. 55

17 Reiner Sans, Änderungen am SGB II werfen neue Probleme auf, in: neue caritas 6/2006, S. 30

18 Vgl. Markus Sievers, Der schwierige Kampf gegen Armutslöhne, in: Frankfurter Rundschau v. 18.8.2006

19 CDU Deutschlands/CSU Landesleitung/SPD Deutschlands (Hrsg.) Gemeinsam für Deutschland, a.a.O., S. 37

\section{Neue Reihe Schriften zur Rechtsbiometrik und zum Alterssicherungssystem}

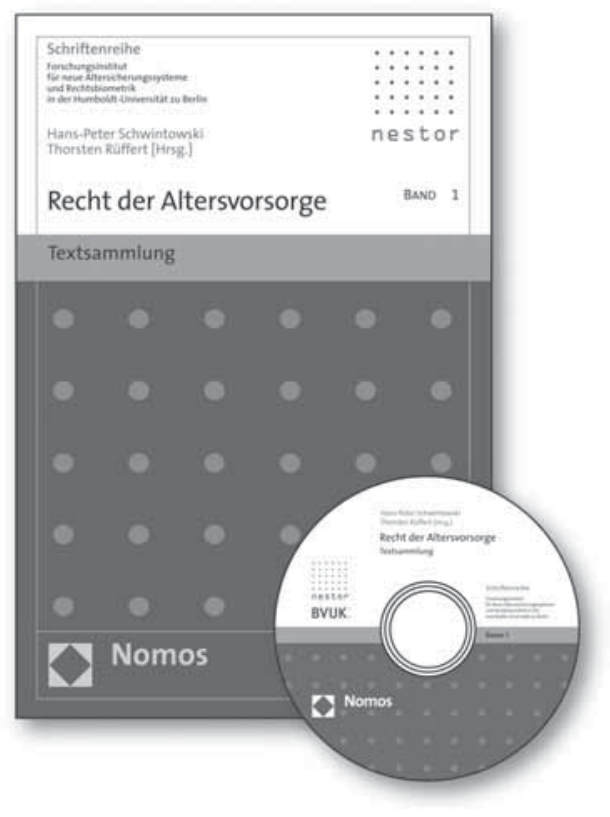

\author{
Recht der Altersvorsorge \\ Textsammlung \\ Herausgegeben von Prof. Dr. Hans-Peter Schwintowski, Humbolt- \\ Universität zu Berlin und Thorsten Rüffert, Humbolt-Universität zu \\ Berlin \\ 2006, 408 S., brosch. mit CD-ROM, 29,-€, ISBN 3-8329-2456-6 \\ (Schriften zur Rechtsbiometrik und zum Alterssicherungssystem, Bd. 1) \\ Auf knapp 400 Seiten enthält die Textsammlung alle wichtige \\ Rechtsvorschriften zur Altersvorsorge. Die beiliegende CD erlaubt \\ darüber hinaus eine vertiefte Recherche zum Recht der Altersvor- \\ sorge. Die Textsammlung richtet sich an alle, die sich von Berufs \\ wegen oder im Rahmen wissenschaftlicher Arbeit mit den Fragen \\ der Altersvorsorge beschäftigen.
}

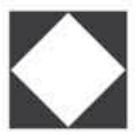

\section{Nomos}

Bitte bestellen Sie bei Ihrer Buchhandlung oder bei: Nomos Verlagsgesellschaft | 76520 Baden-Baden | www.nomos.de 